\title{
Novel iBeacon Placement for Indoor Positioning in IoT
}

\author{
Javad Rezazadeh, Ramprasad Subramanian, Kumbesan Sandrasegaran, \\ Xiaoying Kong, Marjan Moradi, Farshad Khodamoradi
}

\begin{abstract}
Indoor positioning and location estimation inside the buildings is still challenging in Internet of Things (IoT) platform, however GPS signals could successfully solve the outdoor localization problem. A recently introduced RSS-based device, named iBeacon paves the way to estimate the users location inside the buildings. Due to the complexity of indoor RF environments, the positioning accuracy is affected by the placement of the iBeacons. Inadvertently the concept of iBeacon placement for improving the accuracy remains unattended by the current research. This paper provides a comprehensive analysis and experiments on the importance of iBeacon placement, and factors impacting the beacon signal quality. Moreover, we propose a novel beacon placement strategy, Crystal-shape iBeacon Placement (CiP). As another contribution, a customized application for android is developed which is used for recording and analyzing the iBeacon signals. Our proposed placement strategy could achieve $21.7 \%$ higher precision than the existing normal iBeacon placement.
\end{abstract}

Index Terms-iBeacon node, Indoor localization, Indoor Positioning System, IoT.

\section{INTRODUCTION}

$\mathbf{I}$ NDOOR Positioning System (IPS) is one important part of Internet of Things(IoT) where the Location of Everything (LoE) plays an important role to improve most services in IoT [1]. Where are we and how to reach a specific spot inside the big hospital, retail malls, and huge industrial complex? This question has created tremendous interest among academia and industry. Since the successful launch of first Global Positioning System (GPS) satellite, the outdoor localization and navigation could be achieved with greater accuracy compared to indoor environment. Due to signal attenuation caused by various construction materials, the GPS system loses significant power in indoor environment. Further, obtaining the coverage of four satellites in indoor environment is a challenge [2]. Hence, the concept of GPS based positioning was not extended for indoor localization systems. The advent of newer wireless standards such as IEEE 802.15.4, Bluetooth Low Energy (BLE), WiFi, and Radio-frequency identification (RFID) is aiding in developing new indoor positioning technologies. Recently introduced BLE-based device by Apple, iBeacon, enables accurate indoor positioning by providing periodic beacon

Javad Rezazadeh, is with the Faculty of Engineering and Information Technology, University of Technology Sydney, Australia and Tehran North Branch, Islamic Azad University, Tehran, Iran. rezazadeh@ieee.org

Ramprasad Subramanian, Kumbesan Sandrasegaran, Xiaoying Kong are with the Faculty of Engineering and Information Technology, University of Technology Sydney, Sydney, Australia \{Firstname.Lastname\}@uts.edu.au

Marjan Moradi is with the School of Computer Science and Engineering, University of New South Wales and Data61, CSIRO, Sydney, Australia m.moradi@unsw.edu.au

Farshad Khodamoradi is with the Islamic Azad University, Electronic Tehran Branch, Tehran, Iran. E-mail: f.khodamorady@yahoo.com signals[3]. iBeacon technology has been recently applied by the researchers in [4] to adjust the location of smart devices in an indoor environment. This technology is employed to improve indoor localization in the study presented in [5] with the combination of wifi access point. The iBeacon deployment has not been considered while the iBeacon devices were placed, arbitrarily. The same approach has been employed in [6] for iBeacon-based indoor positioning with no efficient iBeacon deployment. The authors improved the location accuracy using Extended Kalman Filter.

In this article, the problem of iBeacon placement for indoor positioning is investigated. We focus on Received Signal Strength Indicator (RSSI) based indoor localization technique with RSSI-based iBeacon motes. The iBeacons install as the reference node to transmit their location information using BLE signals. BLE has broadcasting range of up to $70 \mathrm{~m}$, which makes it an ideal technology for indoor localization. These messages are collected by smart phones, where they can be used for variety of applications such as location detection, push messages for marketing purposes, and prompts.

With the described motivation, the main contributions of this paper are listed as follows:

- We conduct an experimental study to show the problem of RF-based positioning with iBeacons signal quality.

- We introduce a deployment strategy named Crystal-based iBeacon Placement (CiP) for iBeacons employed in indoor positioning task. The placement method is analyzed vertically and horizontally. It experimentally tested and evaluated to validate its efficiency in terms of localization accuracy.

- We develop a customized recording android application for smart phone to collect and analyses RSS data from iBeacons and return queries for every different iBeacons.

The rest of the paper is organized as follows : A review is presented on the existing literatures in Section II. The formulated problem is described in Section III. The proposed iBeacon placement design is presented in Section IV. Experiments, evaluation and analysis are discussed in Section V. Then, the developed application is explained in Section VI. Finally, conclusion is drawn in Section VII.

\section{RELATED WORKS}

Localization, as a crucial service for IoT, is an energydemanding process for both indoor and outdoor scenarios [7]. The localization techniques can be used for Indoor Navigation Systems (INS) to locate objects or people inside a building [8]. Indoor positioning methods can be classified as triangulation, proximity detection and scene analysis [9]. In triangulation technique, geometric features of trigonometry are applied . In 


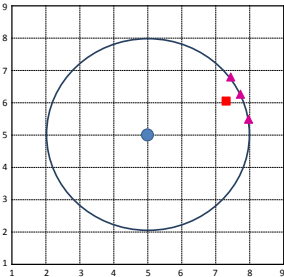

(a) Random placement

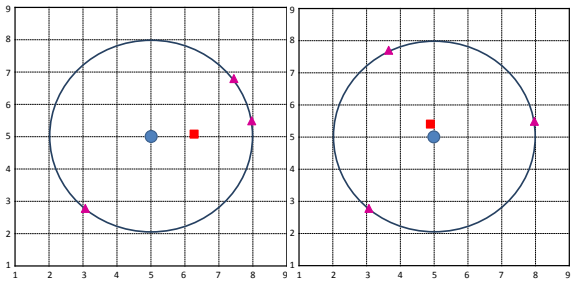

(b) Random placement $\quad$ (c) non-random placement
Fig. 1: Impact of iBeacon placement on localization.

the second methodology of proximity detection, location of target is estimated based on the proximity of known objects. The third methodology of scene analysis is based on two phases: 1) off-line phase and 2) online phase. The off-line phase involves creation of finger printing database. In this database, the intensity of the received signal is collected in the area of interest where localization is intended to be performed. The location of the target is made by matching the received signal with the finger print database. In online phase, a set of Reference Nodes (RN) are installed in the building and these nodes sends out referencing signal for indoor positioning system. The target node that needs to know the location, measures the Received Signal Strength (RSS) from the RN to determine the position. One of the key factors that affect the accuracy of the estimated location of the target node is dependent on beacon placement $[10,11]$. The authors in [4] proposed smartphone inertial sensor-based indoor localization and tracking with corrections applied using iBeacon. In this approach, authors considered important issues such as step detection, walking direction estimation, and initial point estimation. The drift in the walking distance obtained in this approach is re-calibrated using iBeacon. After analysing the drift they authors have proposed extended Kalman filter to re-calibrate the distance. The major shortcoming in this approach is that authors have not proposed where the sensors should be placed. If the sensors are not placed appropriately it may result in high drift and location estimation. In [12], authors propose proximity based localization. This scheme levrages BLE to achieve high level of co-location accuracy. A control theoretic approach namely particle filtering was proposed in [13] to increase the tracking accuracy of indoor environment using iBeacon. The authors have presented experimental results with an error accuracy as low as 0.27 meters. The major disadvantage in this approach is it increases the computational complexity and application of this technique in wider area remains speculative. For large indoor environment location fingerprinting with BLE beacons has been proposed in [14]. The authors have $600 \mathrm{sq} \mathrm{m}$ testbed to position a consumer device. They have demonstrated how to mitigate the high susceptibility of BLE to fast fading. Furthermore, they have investigated the choice of key parameters in a BLE positionign system such as beacon density, transmit power and frequency. They have presented quantitative comparison with WiFi finger printing. But the authors have not considered relationship between the beacon placement and number of beacons required per sq meter. In [15], a beacon based indoor positioning method using extended kalman filter that process data recursively including noise has been proposed. This study recognises the fact that beacon location plays major role in improving the location accuracy but the work of the authors does not expand on it. Another particle filter approach was proposed in [16], in this approach a nonparametric Gaussian Process (GP) model is adopted to describe the relationship between estimated and observed RSS. Then the weights of particles are updates according to the trained GP. The advantage of adopting GP is it considers sensor noise along with multi-path effects, human sheltering effects and so on in the received beacon signals.

\section{Problem Statement}

In this section, we analyse the problem of RF-based indoor positioning system using new Bluetooth Low Energy Transmitter manufactured by Apple, named iBeacon. To the best of our knowledge, it is the first study to investigate and experimentally proof how iBeacon placement is vital for accurate indoor localization. In order to estimate the location of an unknown mobile device, the coordination of 3 iBeacons in its communication range must be exploit [17]. Relying on this principle, we placed 3 iBeacons (with similar technical features) in randomly selected positions in the way of same distance with the receiver (to compensate the impact of RSS) is adjusted. The system model is schematically depicted in Figure 1 where three purple triangles show iBeacons and the receiver node (the blue circle) is in the center. As it is obvious from the figure, the difference between the estimated location (red rectangle) and actual location (blue circle) through different placement is changed. In Figures 1(a) and 1(b) randomlyplaced iBeacons provide less location precision than Figure 1(c). The localization error is calculated by measuring the distance between the real location of a node and its estimated location. Indeed, in this basic experiment we have conducted same localization algorithm, Accuracy-Priority Trilateration (APT) [11] with the same type of beacon device, iBeacon [3] to just evaluate the impact of a carefully selected iBeacon placement to achieve precise indoor location estimation.

To conclude, the localization error in an indoor environment is a function of factors:

$$
L_{e}=f(M, A, B, P)
$$

where $M$ represents the mapping error, $A$ denotes the error due to the localization algorithm, $B$ shows technical features of the beacon (in our article the type of nodes used is iBeacon), and $P$ denotes iBeacon placement.

\section{Proposed Method: Crystal-Shape iBeacon PlaCEMENT (CIP)}

As discussed in Section III, analysis of the placement for iBeacons, as the reference node for indoor positioning, is a should-do task to achieve maximum precision. In this section, we present our proposed placement methodology for iBeacons in an indoor environment for mobile devices positioning. 


\section{A. Desired height}

The placement scheme must meet the critical positioning requirements such as localization coverage, success and accuracy. Let's assume $L_{i B_{N r}}$ shows the location of $N r$ number of iBeacons installed for a specific Region of Interest (RoI). These $N r$ iBeacons should put in the way that:

- Every position (for users' smart phones) in the RoI must ensure to be covered by at least 3 different iBeacons (localization coverage).

- $L_{i B_{N r}}$ for the 3 contribiuters iBeacon should be noncollinear (localization success and accuracy).

- The shortest possible distance between $L_{i B_{N r}} \mathrm{~s}$ and receiver devices need to be provided (localization precision).

- The optimal number of iBeacons must be deployed for maximum coverage (efficient network cost).

In the indoor layout, all users must be serviced from the height of a wheelchair $(60 \mathrm{~cm})$ to an average man as tall as $175 \mathrm{~cm}$. The area between, seems as a candidate area for received signals by iBeacons. Figure 2 denotes different possible deployment through the explained ideal space.

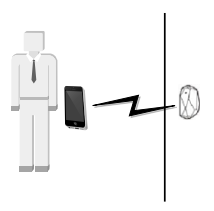

(a) Ideal Location

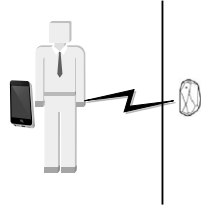

(b) Impact of $\mathrm{BB}$

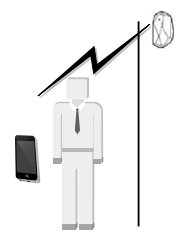

(c) BB handling
Fig. 2: : iBeacon placement strategy to negate Body Blockage (BB)

As could be seen in Figure 2(a), the placement is considered at the heigh of $1 \mathrm{~m}$ which is horizontally opposed to the mobile devices in users' hand. In spite of the strong received signal quality in the ideal height, this placement is most likely to faced with the problem of Body Blockage, $B B$, (Figure 2(b)). None-Line of Sight (NLOS) path causes to establish poor communication or even no received signal. In order to overcome $B B$ issue, iBeacons should be placed over the height of $175 \mathrm{~cm}$ while the ideal area must be covered by the iBeacon signals. Here, we conduct an experimental measurement to figure out which height is efficient for placement in the targeted scenario. Figure 3 plots the measured RSS values where the height of the iBeacon is changing from $190 \mathrm{~cm}$ to $240 \mathrm{~cm}$ by $10 \mathrm{~cm}$ for each experiment. In this figure, $x$ axis shows the distance between iBeacon and the receiver device while $y$ axis is its corresponding $R S S$ values. It enlarged from $1 \mathrm{~m}$ to $5 \mathrm{~m}$ to investigate the received power trend in different distances. The significant finding from the experiment is that the higher RSS provides at the height of $210 \mathrm{~cm}$ over different distances. Consequently, we consider this value as the best desired height, $h_{\text {iBeacon }}$, for iBeacon placement.

To validate the efficiency of the desired height, we measure RSS value in three different scenarios plotted in Figure 2. The measured value are reported by Figure 4 . As observed from Figure 4(a), BB scenario has least RSS measurement. Though, the proposed vertical strategy placement could enhance the

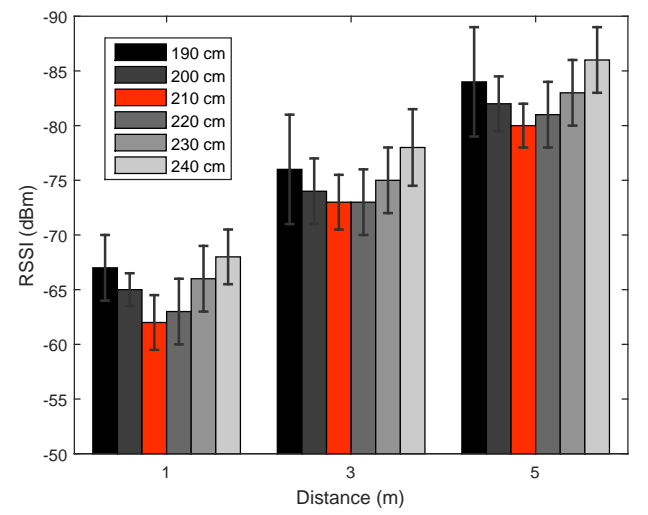

Fig. 3: RSS comparison in different heights $(190-240 \mathrm{~cm})$.

value, significantly. Figure 4(b) reports numerical value of average RSS in three different scenario. It shows how the proposed vertical placement (brown bar) could overcome the $\mathrm{BB}$ issue and improve RSS most equal to the scenario without BB (black bar). Figure 4(c) plots the collected RSS for 40 samples of signals. Proposed vertical placement provides almost similar values of measured RSS. It leads to offer more reliable and accurate positioning based on the stable RSS values.

\section{B. Horizontal placement}

The next step is to place the nodes, horizontally, in a way that every position is covered by 3 different non-colinear iBeacons to achieve highly accurate position estimation with minimum possible number of iBeacons. We figured out the desired height of the iBeacons $h_{\text {iBeacon }}$ in Section IV-A, thus $3 D$ deployment is mapped to $2 D$. Let's consider the horizontal deployment area as $\tau$. Thus:

$$
L_{B i}=\left\{L_{B 1}, \ldots, L_{B N r}\right\} \in \tau
$$

where $L_{B i}$ is the set of coordinations inside the deployment plane to place $N r$ numbers of iBeacons. Initially, we put first iBeacon on the location of $L_{B 1}$ and labeled it as $A$, as seen in Figure 5. The coverage area of the iBeacon is ideally formed as a circle at center $A$ and radius $R$, the iBeacon communication range. Its coverage area, $\Phi$, could be formulated as:

$$
\Phi_{B 1}=x_{A}^{2}+y_{A}^{2}=R^{2}
$$

Now, the second iBeacon must be placed in a position $L_{B 2}$, where:

$$
L_{B 2}= \begin{cases}L_{B 2} \in \Phi_{B 1} & \text { (i) } \\ \max \left\{\Phi_{B 1} \cup \Phi_{B 2}\right\} & \text { (ii) } \\ \max \left\{\Phi_{B 1} \cap \Phi_{B 2}\right\} & \text { (iii) }\end{cases}
$$

These conditions are required to guarantee:

i. Enabling User's devices to receive sufficient numbers of iBeacon messages for localization.

ii. Deploying least possible number of iBeacons.

iii. Maximizing covering $(\max )$ area by the iBeacon. 


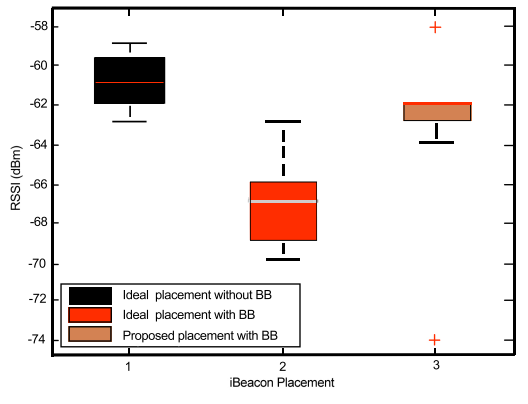

(a) Box-and-whisker plot for RSS

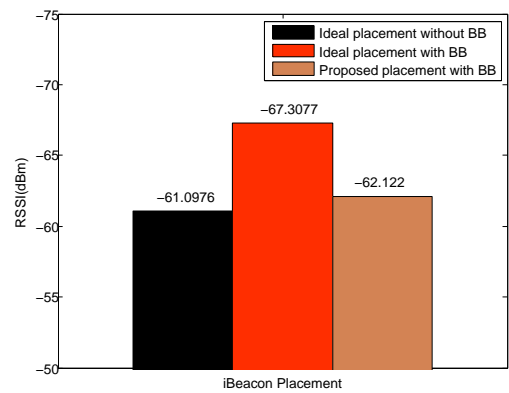

(b) Numerical Results for average RSS

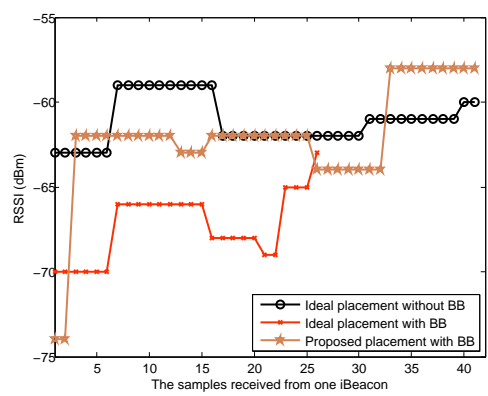

(c) RSS velues for all sample signals

Fig. 4: Experimental results of iBeacon vertical placement in different scenarios.

The conditions listed in Equation 4 meet if and only if the second iBeacon lies on the circle centered at $A$ (first iBeacon location). It conducts to specify the coordination of the second iBeacon, $L_{B 2}$ as:

$$
L_{B 2}=\left(x_{A}-x_{B}\right)^{2}+\left(y_{A}-y_{B}\right)^{2}=R^{2}
$$

We pick $B$ for $L_{B 2}$ which is shown in Figure 5 .

Next, we have to place the third iBeacon in order to provide sufficient number of beacon messages and complete localization task. The listed conditions in 4 should be satisfied to achieve the objectives of the proposed placement strategy. Thus the equation is updated with the condition to specify $L_{B 3}$ as follow:

$$
L_{B 3}= \begin{cases}L_{B 3} \in\left\{\Phi_{B 1} \cap \Phi_{B 2}\right\} & \text { (I) } \\ \max \left\{\Phi_{B 1} \cup \Phi_{B 2} \cup \Phi_{B 3}\right\} & \text { (II) } \\ \max \left\{\Phi_{B 1} \cap \Phi_{B 2} \cap \Phi_{B 3}\right\} & \text { (III) }\end{cases}
$$

It could be observed form Figure 5 that the required conditions in Equation 6 is meet by 2 points: $C$ and $G$. Let's pick $C$ as the position of $L_{B 3}$. The distance between every pair of the positions, $A B, A C$, and $B C$ is equal (with $R$ ). It is concluded that:

$$
\overline{\mathrm{AB}}=\overline{\mathrm{AC}}=\overline{\mathrm{BC}}=R \Rightarrow \triangle A B C \text { is equilateral }
$$

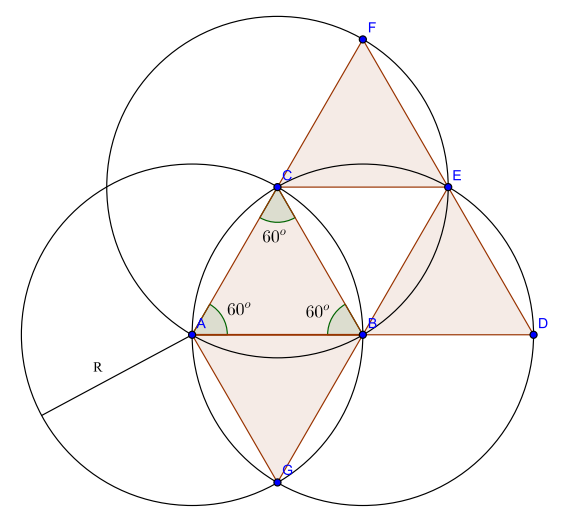

Fig. 5: Crystal-shape iBeacon Placement (CiP)

Thus, best possible deployment for iBeacons in indoor positioning task is an equilateral triangle. To extend the coverage area of indoor positioning, we need to place more iBeacons in adjacent position of the deployed equilateral triangle. The extended design forms a crystal shape which is inspired us to name the model as Crystal iBeacon Placement, "CiP".

It should be considered that the proposed iBeacon placement, $\mathrm{CiP}$, is conducted by the mathematical relations formulated in Equation (4-6). The derived equations could satisfy the efficient arrangement of the iBeacons for accuracy improvement of indoor positioning. In contrast with the randomly placement of the iBeacons, the proposed mathematical conditions guarantee the shortest possible distance among three devices which is required for localization and consequently offer more accurate position estimation.

\section{IMPLEMENTATION AND EVALUATION}

In this section, we describe CiP implementation, the experimental test setup and the performance evaluation of CiP placement strategy. We conduct our experiments in Level-8, University of Technology Sydney (UTS) Engineering Building as the floor plan is shown in Figure 6.

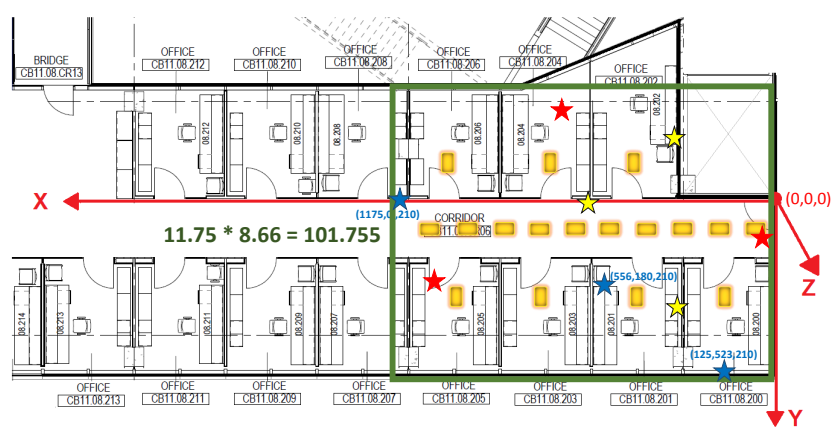

Fig. 6: Experimental platform: (blue: arbitrary placement, yellow: $Z$ placement, red: $C i P$, orange: signal measurement locations)

Testing was carried out as a comparison between three different iBeacon placement techniques. The first placement is the usual one suggested in Estimote website which the iBeacons are placed arbitrarily such as research done for iBeacon-based indoor positioning [5]. The second deployment strategy is $Z$ placement method presented in [11]. The method, though, originally designed for traveling by mobile beaconassisted localization. The path meet the localization requirement, hence could be competitive placement strategy for 


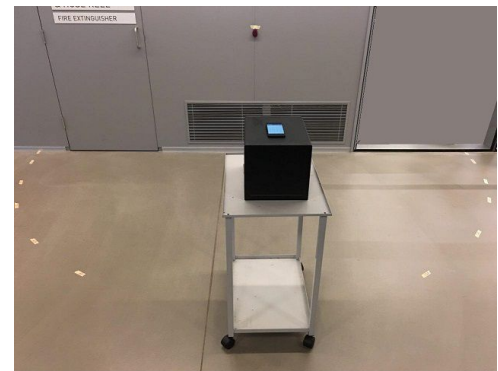

(a) iBeacon beam measurement setup

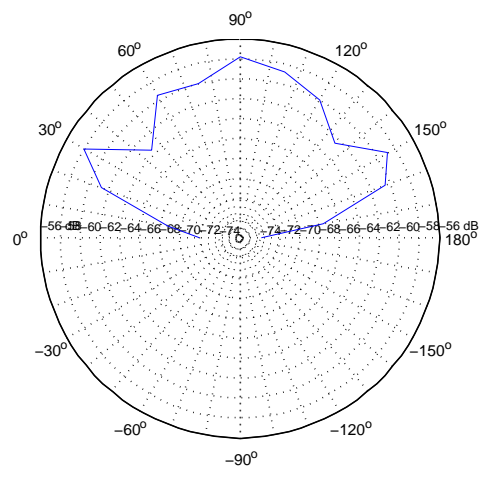

(b) iBeacon beam in horizontal direction

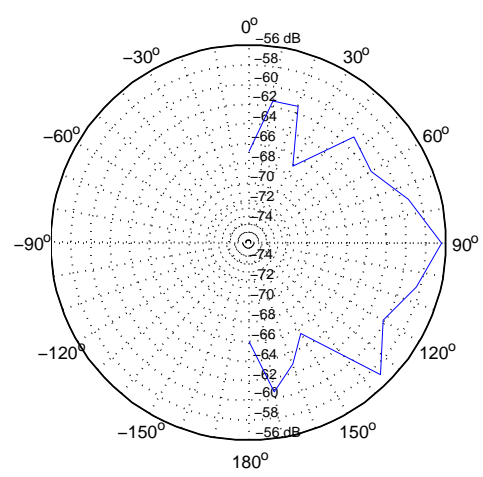

(c) iBeacon beam in vertical direction

Fig. 7: The radiation pattern of iBeacon in horizontal and vertical direction.

iBeacons employed in positioning purpose. $\mathrm{CiP}$ is evaluated where the iBeacons are placed to form an equilateral triangle.

In this test, the iBeacon signal interval used set as $1285 \mathrm{~ms}$ and broadcasting power is $-12 \mathrm{dBm}$. This is the default documentary settings for iBeacon released by the company.

\section{A. Experimental Setup}

Here, we describe the testbed setup and analyze the measured signal in respect with the iBeacon orientation and its radiation pattern. As it is shown by Figure 7(a), we conducted our signal collecting experiment in an indoor setting. The iBeacon is installed on the wall at the height of $1 \mathrm{~m}$, in order to match the targeted scenario of indoor positioning. We have used iBeacons made by Estimote in our project. The iBeacon contains flexible powerful multiprotocol System-ona-chip (Soc) with an nRF51822 chip which is built around a 32-bit ARM Cortex M0 CPU with $256 \mathrm{kB} / 128 \mathrm{kB}$ flash and $32 \mathrm{kB} / 16 \mathrm{kB}$ RAM. The iBeacon simply transmits Bluetooth packets with identification data so called advertisements that contain four parts: 1- MAC address 2- Universally unique identifier (UUID), common for a single deployment at a venue. 3- Major number, designated for dividing the beacon sets into smaller segments. 4- Minor number, designated for dividing the segments into smaller subsegments [5]. For receiver side, a mobile phone is placed over a trolley at the height of $1 \mathrm{~m}$ from the ground and at $2 m$ distance from iBeacon. Non-obstructed direct path condition is ensured. We perform measurement at various angles starting from $\theta=90^{\circ}$ on either side, where the distance between iBeacon and receiver was kept constant. $\theta$ is the angle between the line from receiver to transmitter and the wall in which the iBeacon is installed over. The labelled position placed on Figure 7(a) are the positions of performing signal measurement. Time duration for signal collecting is $20 s$ at each labelled position. The measurement was made at various values for $\theta$ such as $75^{\circ}, 60^{\circ}, 45^{\circ}, 30^{\circ}, 20^{\circ}, 10^{\circ}$, and $0^{\circ}$ on the right side and $105^{\circ}, 120^{\circ}, 135^{\circ}, 150^{\circ}, 160^{\circ}, 170^{\circ}$, and $180^{\circ}$, on the other side.

For the power plot, Figure 7(b) and 7(c) shows horizontal and vertical pattern of received signal strength in a polar coordinate system. An obvious investigation is the maximum received power at the angle of $\theta=90^{\circ}$ for both vertical and horizontal directions. Despite the fact of omnidirectional antenna in iBeacon, the received signal strength has some fluctuations. Generating a perfect omni directional pattern is experimentally impossible and our measurements show it on the figures. One reason is that the radiation pattern tends to be affected by the environment outside the antenna, such as enclosures on which the antenna is mounted, and objects around the enclosures. This variation is observed from Figure 7 (b) at the angles of $60^{\circ}$ to $30^{\circ}$, and $120^{\circ}$ to $150^{\circ}$ on horizontal direction. Further, vertical direction has experienced the signal strength fluctuations around the angles of $20^{\circ}$ to $50^{\circ}$ and $140^{\circ}$ to $170^{\circ}$ which is plotted by Figure 7 (c).

\section{B. Signal Quality Measurement}

The previously discussed measurement results deduced the signal strength variation in respect with the iBeacon orientation in a line of sight condition. In this section, the impact of two main factors are experimentally evaluated, surrounding materials and the distance. The findings are promising to come up with the best possible deployment of iBeacon for targeted indoor navigation application. The results are plotted in Figure 8.

In order to analyze the effect of building materials on the propagation loss, we evaluate the iBeacon signal quality in an indoor environment made up of different composite. Figure 8(a) shows the testbed area of various building materials such as an area with glass walls, corridor with glass and cement walls, corridor with only cement walls, and a wide area. Figure 8(b) plots the measured signal strength received through these environments. The results indicate that the glass area imposes higher propagation loss compared with other materials in a line of sight condition. The trend is valid over different distances, just decreases the RSS value in farther. In a blocked communication path or NLOS condition, the quality of the measured signal is significantly dropped and it is getting worse over the long distances. The red color bar indicates the average provided RSS value for a direct path area through the building with different materials.

Next, we consider the received power over various distances from iBeacon. Let us start with Friis transmission which shows the received power as inversely proportional to distance. The 


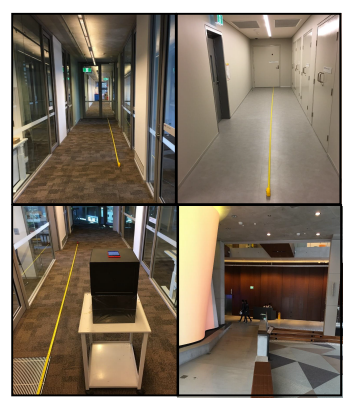

(a) Testbed conducted in UTS

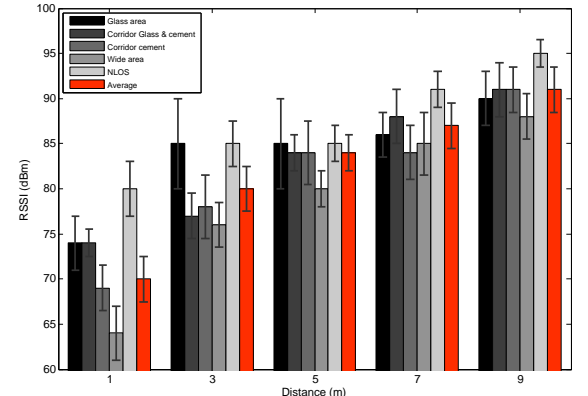

(b) Measured RSS in different materials and distance

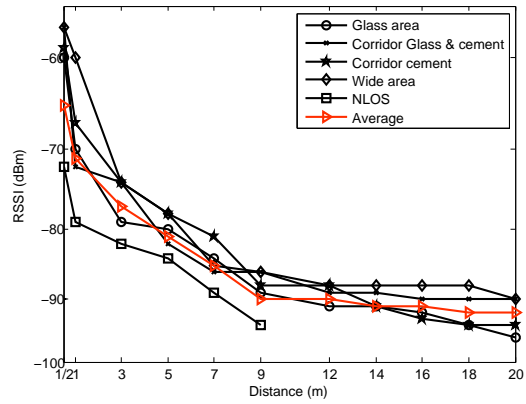

(c) RSS vs. Distance for different materials

Fig. 8: Signal quality measurement vs distance in various environment

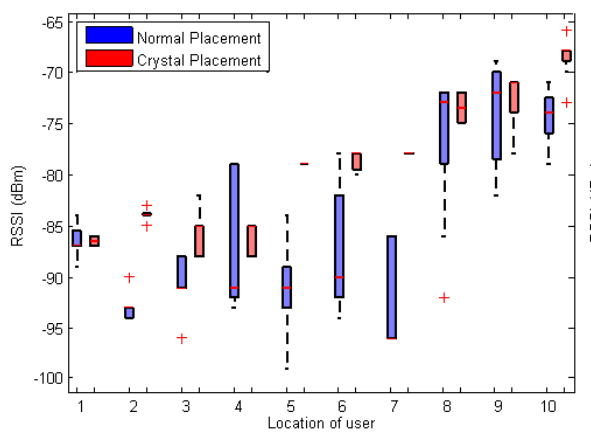

(a) Box-and-whisker plot for iBeacon 1

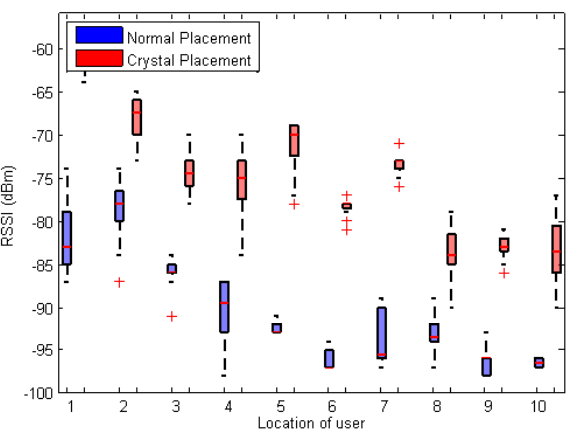

(b) Box-and-whisker plot for iBeacon 2

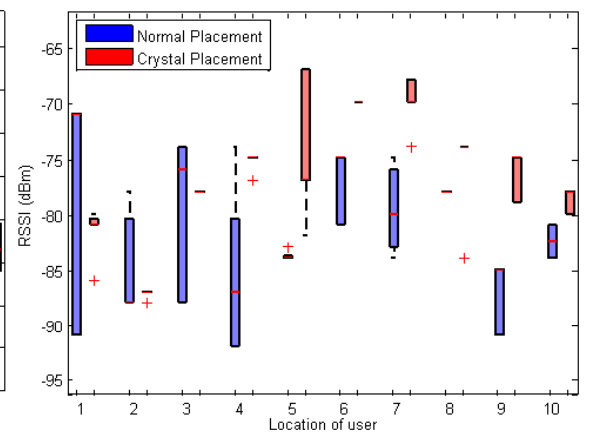

(c) Box-and-whisker plot for iBeacon 3

Fig. 9: Comparison of signal overlapping between normal placement and $\mathrm{CiP}$ for three iBeacons placed in different positions

measured values of RSS which plotted in Figure 8(c) clearly confirm this concept. In this figure, the reverse impact of distance is evaluated regarding with the building materials as well. The significant finding is the constant RSS value in spite of larger distances in all environment. As seen in the figure, signal power is decreasing from $1 \mathrm{~m}$ to $9 \mathrm{~m}$, but it stays constant regardless of farther distances. This behavior reveals that the collected signal from greater than a threshold distance is inadequate for indoor positioning. This is due to the necessity of calculating the distance based on RSS value. This distance threshold is valid for NLOS scenario as well in which no signal is received. To conclude, the signal received $9 m$ and farther than that, is not accurate enough for indoor navigation.

\section{C. iBeacon placement and Received Signal Strength}

iBeacon placement indoor navigation system is a novel research area and none of the studies report experimental evaluation in this regard. In this research, we present preliminary experiments to verify the superiority of our proposed iBeacon deployment, CiP. Figure 9 plots Box-and-Whisker diagram where receiver's location and its corresponding RSS values are represented by $x$ and $y$ axis, respectively. Each subfigure shows RSS values collected from a specific iBeacon, namely iBeacon 1, 2, and 3. In this figure, 2 different iBeacon deployments are tested, normal placement (blue bar) [5] and proposed CiP (red bar).

The experiment launched with signal strength measurement from the origin point $((0,0,0)$ for every meter interval. The first deduction of the figure is that $\mathrm{CiP}$ deployment enables to provide more positioning precision than normal placement. By looking at the figure, it could be find that the collected RSS from iBeacons with normal deployment reports wider variety of values than $\mathrm{CiP}$ placement. It causes to calculate inaccurate distances of transmitter- receiver pair. Moreover, the measured RSS values in the normal experiment have greater overlap in comparison with CiP placement. For instance, received power equals with $-85 \mathrm{dBm}$ from iBeacon 3 has been experienced by five different locations e.g., location numbers of 1,2,3,4, and 9. This behavior makes confusion for distance calculation.

The performance of CiP is compared with $Z$ placement strategy which proposed in [11]. The measured RSS from three iBeacons with different arrangements were averaged and plotted by Figure 10. The figure shows a box- and- whisker plot for RSS values of 3 iBeacons in accordance with three different deployments. The most critical interpretation of the figure is the higher received power from $\mathrm{CiP}$ placement than 2 other schemes. As it is obvious from the figure, the taller boxes of normal and Z placements, suggests a difference on the received power compared with $\mathrm{CiP}$ placement. This issue has a direct impact on the precision of the estimated position due to the fact of contributing signals with a significant difference in RSS value. 


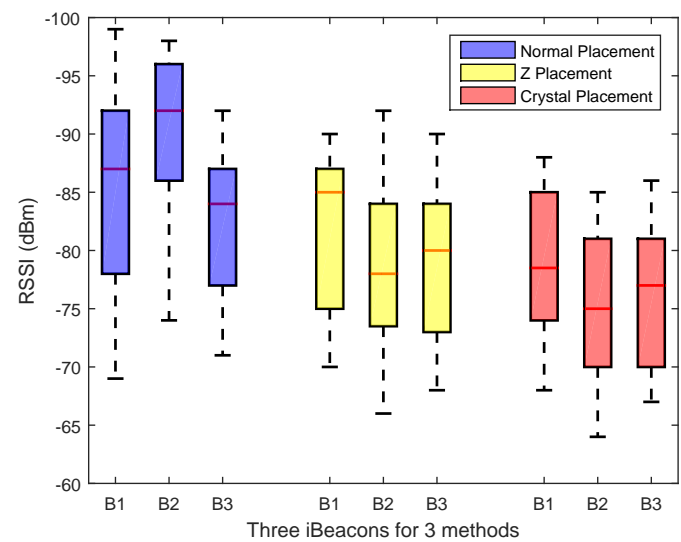

Fig. 10: Box-and-whisker plot for average RSS in various placement

\section{Indoor Positioning Accuracy}

In this section, we test and verify the effectiveness and efficiency of CiP in the estimation of device position. Hence, the measured data collected from 3 different deployment methods (e.g., normal, z-path, and CiP) were exploited to calculate the location of users' devices inside the building. We exploit Weighted Centroid Localization (WCL) technique for RSSbased positioning purpose which has extensively described in our previous research, $[11,17]$. WCL averages the coordinates of all received signals from $N_{r}$ iBeacons. Let's assume the calculated position, $p_{\left(s_{i}\right)}$, is formulated as follows:

$$
p_{\left(s_{i}\right)}=\frac{\sum_{j=1}^{N_{r}}\left(w_{i j} . b_{j}(x, y)\right)}{\sum_{j=1}^{N_{r}} w_{i j}}
$$

where $b_{j}(x, y)$ denotes the location of iBeacon. Each signal is weighted by the received power using weight function, $w_{i j}$. $w_{i j}$ is replaced by $R S S_{i j}$ and the final equation is :

$$
p_{\left(s_{i}\right)}=\frac{\sum_{j=1}^{N_{r}}\left(R S S_{i j} . b_{j}(x, y)\right)}{\sum_{j=1}^{N_{r}} R S S_{i j}}
$$

Location estimation accuracy for the compared iBeacon placement methods and $\mathrm{CiP}$, are reported in Figure 11. Accuracy, the difference between the estimated and the actual location, is a critical metric for validating the performance efficiency of the placement technique. CiP provides lower location error than $\mathrm{Z}$ and normal iBeacon placements because of higher quality of the received signals. The higher the RSS, the more accurate the localization. CiP could successfully enhance the accuracy of indoor localization by $21.6 \%$.

\section{IBEACON Signal ReCording APP}

One of the contributions of this research is developing a customized application for android smart phones. This app can collect the propagated signals from the iBeacons, recorde them in a ".csv" file format and share the file by email, telegram and text message. Currently, the existing app developed for iBeacon such as Estimote, Beacon Scan, Indoor, and Dart is not efficient for localization while they are unable to record signals in the desired table format. Our developed app records

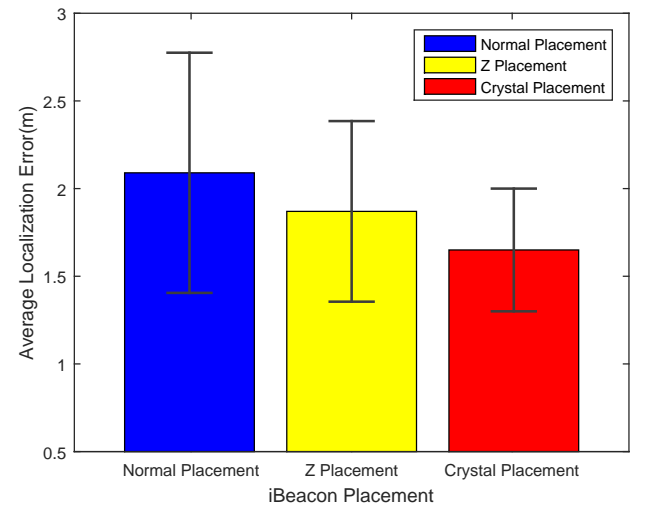

Fig. 11: Average localization error calculated by WCL method

the data in six typical fields: beacon ID, signal receive time, major/minor of the UUID, and RSSI in $\mathrm{dBm}$ to enable post processing. This app is designed to record signals in two different modes, momentary and continuous. In momentary mode, the app records the signals that are received at the moment when "stop" button is clicked and the values are saved by entering the label information. In continuous mode, app records all the signals received during a period of time. At first, a number is inserted as duration time and "start" button is clicked to start the recording process. Then app requests the user to insert the label information and the recording is continuously performed until finish the time. Figure 12 illustrates the User Interface (UI) of the app.
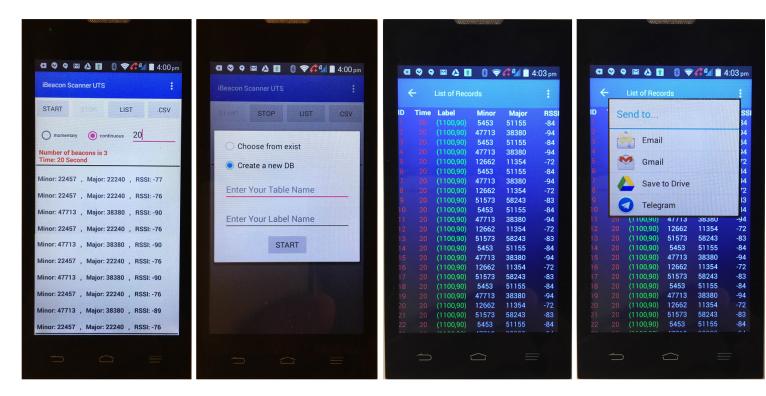

Fig. 12: Customized android application UI interface

\section{CONCLUSION}

In Internet of Things (IoT) technologies, iBeacom motes, as a promising infrastructure of indoor localization, requires more research and evaluation. In this paper, impact of iBeacon placement for localization accuracy was considered. We experimentally evaluated the problem of RF-based positioning with iBeacon signal quality. Then, we introduced Crystalbased iBeacon Placement (CiP) for iBeacons employed in indoor positioning. The placement method has been analyzed vertically and horizontally. It experimentally was tested and evaluated to validate its efficiency and yielded $21.16 \%$ improvement in terms of accuracy. Moreover, a customized android application was developed to collect and measure the signal from iBeacons, timely and efficiently. As future direction, we are planning to evaluate an Indoor Navigation System based on CiP idea and a machine learning method. 


\section{REFERENCES}

[1] J. Rezazadeh, K. Sandrasegaran, and X. Kong, "A location-based smart shopping system with iot technology," in IEEE 4th World Forum on Internet of Things (WF-IoT), 2018.

[2] S. He and S. H. G. Chan, "Wi-fi fingerprint-based indoor positioning: Recent advances and comparisons," IEEE Communications Surveys Tutorials, vol. 18, 2016.

[3] P. Martin, B.-J. Ho, N. Grupen, S. Muñoz, and M. Srivastava, "An ibeacon primer for indoor localization: Demo abstract," in the 1st ACM Conference on Embedded Systems for Energy-Efficient Buildings, 2014.

[4] Z. Chen, Q. Zhu, and Y. C. Soh, "Smartphone inertial sensor-based indoor localization and tracking with ibeacon corrections," IEEE Transactions on Industrial Informatics, vol. 12, no. 4, pp. 1540-1549, 2016.

[5] F. M. Pavel Kriz and T. Kozel, "Improving indoor localization using bluetooth low energy beacons," Mobile Information Systems, vol. 2016, 2016.

[6] L. Seoung-Hyeon, L. Il-Kwan, and L. Jae, "Method for improving indoor positioning accuracy using extended kalman filter," Mobile Information Systems, 2016.

[7] J. Rezazadeh, M. Moradi, K. Sandrasegaran, and R. Farahbakhsh, "Transmission power adjustment scheme for mobile beacon-assisted sensor localization," IEEE Transactions on Industrial Informatics, 2018.

[8] M. Sattarian, J. Rezazadeh, R. Farahbakhsh, and A. Bagheri, "Indoor navigation systems based on data mining techniques in internet of things: a survey," Wireless Networks, 2018.

[9] H. Liu, H. Darabi, P. Banerjee, and J. Liu, "Survey of wireless indoor positioning techniques and systems," IEEE Transactions on Systems, Man, and Cybernetics, Part C, vol. 37, no. 6, pp. 1067-1080, 2007.

[10] A. Yassin, Y. Nasser, M. Awad, and A. Al-Dubai, "Recent advances in indoor localization: A survey on theoretical approaches and applications," IEEE Communications Surveys Tutorials, 2017.

[11] J. Rezazadeh, M. Moradi, A. S. Ismail, and E. Dutkiewicz, "Superior path planning mechanism for mobile beacon-assisted localization in wireless sensor networks," Sensors Journal, IEEE, vol. 14, 2014.

[12] P. M. Varela and T. O. Ohtsuki, "Discovering co-located walking groups of people using ibeacon technology," IEEE Access, vol. 4, pp. 6591-6601, 2016.

[13] F. Zafari and I. Papapanagiotou, "Enhancing ibeacon based micro-location with particle filtering," in IEEE Global Communications Conference, 2015.

[14] R. Faragher and R. Harle, "Location fingerprinting with bluetooth low energy beacons," IEEE Journal on Selected Areas in Communications, vol. 33, 2015.

[15] L. Seoung Hyeon and L. Jae Kwang, "Method for improving indoor positioning accuracy using extended kalman filte," Mobile Information Systems, vol. 16, 2016.

[16] Q. S. Y. Gu and M. Ma, "Using ibeacons for trajectory initialization and calibration in foot-mounted inertial pedestrian positioning systems," in International Confer- ence on Indoor Positioning and Indoor Navigation, 2016.

[17] J. Rezazadeh, M. Moradi, A. S. Ismail, and E. Dutkiewicz, "Impact of static trajectories on localization in wireless sensor networks," Wirel. Netw., vol. 21, no. 3, pp. 809-827, 2015.

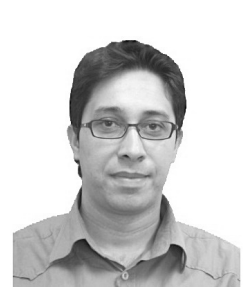

Javad Rezazadeh is a researcher at University of Technology, Sydney (UTS) in Australia and also an assistant professor at the Azad University of North Tehran Branch in Iran. He holds a PhD in Wireless Communications from University Technology Malaysia while being received Academic Excellent Student Award for the top 1 postgraduate in the year 2014. His research interests include localization systems, Internet of Things (IoT) and machine learning.

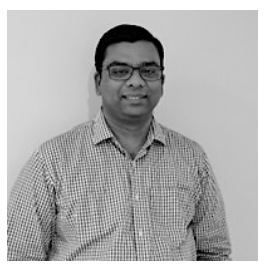

Ramprasad Subramanian is a senior IoT engineer at National Narrowband Network Communications company, Sydney, Australia. He received the Master of Engineering (M.Eng.) by research in telecommunications from University of Technology Sydney (UTS), Australia in 2017. His research interests include IoT, data probing, mobile computing and wireless communications.

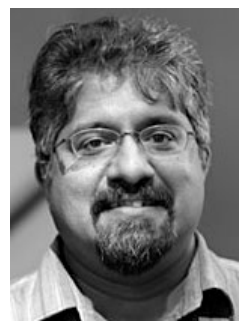

Kumbesan Sandrasegaran is an Associate Professor at University of Technology, Sydney. He holds a $\mathrm{Ph} . \mathrm{D}$. in Electrical Engineering from McGill University, Canada, 1994. His current research work focuses on two main areas radio resource management in mobile networks, and engineering of remote monitoring systems for novel applications with industry through the use of embedded systems, sensors and communications systems.

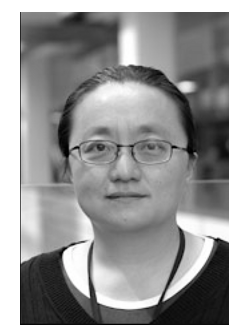

Xiaoying Kong is currently a senior lecturer at University of Technology, Sydney. She received her Bachelor of Engineering and Master of Engineering in control engineering from Beijing University of Aeronautics and Astronautics in 1986 and 1989 respectively. She received $\mathrm{PhD}$ degree in mechatronic engineering from the University of Sydney in 2000. Her research area includes inertial navigation systems, indoor positioning, multi sensor fusion, trajectory planning, and web engineering.

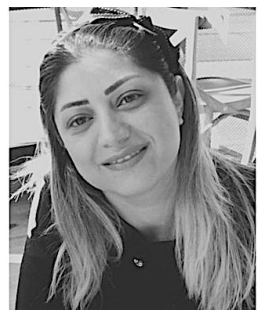

Marjan Moradi is currently pursuing the Ph.D. degree in computer science at the University New South Walse (UNSW) and also researcher at Data61, CSIRO, Australia. She received the M.Sc. degree by research in computer science from University Technology Malaysia (UTM) while being received Excellent Researcher Award in 2013. Her research interests include mobile networks, Millimeter wave networking, IoT and machine learning.

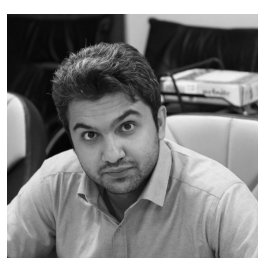

Farshad Khodamoradi is a chief technology officer at $\mathrm{CtrlTech}$. He is currently pursuing the M.Sc degree in software engineering at Islamic Azad University, Electronic Tehran Branch, Iran. He has experience of working in industrial program development and extensive skills in JAVA, Android, Server Side, PHP, DB and Web Design. His research interests include software engineering and systems modeling. 